\title{
小麦叶片氮素状况与光谱特性的相关性研究
}

\author{
薛利红 曹卫星* 罗卫红 张 宪 \\ (南京农业大学农业部作物生长调控重点开放实验室，南京 210095)
}

\begin{abstract}
摘 要 系统分析了不同时相下两个小麦 (Triticum aestivium) 品种叶片含氮量及叶片氮积累量与冠层光谱反射特征 的关系。结果表明，随施氮水平的增加，小麦冠层在可见光区的反射率逐渐降低，而近红外波段的反射率逐渐升 高。小麦叶片氮素状况与比值指数或归一化指数显著相关, 两个品种表现极为一致, 可以用一个指数方程来拟合。 分阶段建模并没有提高模型的精度，因此可以建立一个适用于整个生育时期的通用氮素诊断方程。叶片含氮量同 光谱指数在整个生育期内的关系要优于叶片氮积累量的, 其中, 与叶片含氮量关系最佳的指数为红波段 $(660 \mathrm{~nm})$ 和 蓝波段 $(460 \mathrm{~nm})$ 的组合 $\left(R^{2}>0.80\right)$;与叶片氮积累量关系最佳的光谱指数为中红外波段 (1 $\left.220 \mathrm{~nm}\right)$ 与红波段 (660 $\mathrm{nm})$ 的组合 $\left(R^{2}>0.62\right)$ 。

关键词 小麦 叶片氮素状况 光谱特性 比值指数 归一化指数 回归模型

\section{CORRELATION BETWEEN LEAF NITROGEN STATUS AND CANOPY SPECTRAL CHARACTERISTICS IN WHEAT}

\author{
XUE Li-Hong CAO Wei-Xing ${ }^{*} \quad$ LUO Wei-Hong and ZHANG Xian \\ ( Key Laboratory of Crop Growth Regulation, Ministry of Agriculture , Nanjing Agricultural University , Nanjing 210095 , China)
}

Abstract Non-destructive monitoring and diagnosis of plant nitrogen $(\mathrm{N})$ status is necessary for precision $\mathrm{N}$ management. The present study was conducted to determine if canopy reflectance could be used to evaluate leaf N status in wheat ( Triticum aestivium) of two cultivars ' Huaimai 18' and' Xuzhou 26' . Ground-based canopy spectral reflectance, leaf $\mathrm{N}$ concentration and leaf $\mathrm{N}$ accumulation were measured at seven growth stages (jointing , booting, heading, anthesis , initial filling, mid-filling and late filling) under four different treatments of $\mathrm{N}$ fertilization $\left(0,12,21\right.$ and $\left.30 \mathrm{~g} \mathrm{~N} \mathrm{~m}^{-2}\right)$. Analyses were made on the relationships of seasonal canopy spectral reflectance, ratio indices $\left(R\left(\lambda_{1}, \lambda_{2}\right)=\rho_{\lambda_{1}} / \rho_{\lambda_{2}}\right)$ and normalized difference indices $\left(N D\left(\lambda_{1}\right.\right.$,

$\left.\left.\lambda_{2}\right)=\frac{\left|\rho_{\lambda_{1}}-\rho_{\lambda_{2}}\right|}{\rho_{\lambda_{1}}+\rho_{\lambda_{2}}}\right)$ to leaf $N$ concentration and $N$ accumulation in wheat under different $N$ treatments at different growth stages and during the whole growing season. The results showed that canopy spectral reflectance in the near infrared increased with increasing $\mathrm{N}$ content, whereas reflectance in the visible band decreased. Leaf $\mathrm{N}$ status was significantly correlated with the ratio or normalized difference indices in both cultivars. Regression models of leaf $\mathrm{N}$ status to spectral indices based on single growth stages did not improve the prediction over whole growth cycle ; hence, a single regression model based on the whole growth cycle should be adequate for diagnosing $\mathrm{N}$ status in wheat plants. The relationships between leaf $\mathrm{N}$ concentration and spectral indices were better than those of leaf $\mathrm{N}$ accumulation. The best indices were the combination of a MIR (mid-infrared) band $(1220 \mathrm{~nm})$ and red band $(660 \mathrm{~nm})$ for leaf $\mathrm{N}$ concentration $\left(L N C=3.8945 \times R(660,460)^{-1.0287}, R^{2}=\right.$ $\left.0.8342 ; L N C=3.9187 \times e^{-2.1796 N D(660460)}, R^{2}=0.8352\right)$ and the combination of the red band (660 $\mathrm{nm})$ and blue band $(460 \mathrm{~nm})$ for leaf $\mathrm{N}$ accumulation $L N A=1.55 \times e^{0.1153 R(1220,660)}, R^{2}=0.7242 ; L N A$ $\left.=0.2194 \times e^{4.0914 N D(1220,660)}, R^{2}=0.6299\right)$. These results indicate that canopy spectral reflectance can be used to non-destructively monitor leaf $\mathrm{N}$ status in wheat plants.

Key words Wheat, Leaf nitrogen status, Spectral characteristics, Ratio index , Normalized difference index , Regression model

氮肥是当今农业生产上用量最大且难以准确定 量的一种肥料,也是生产中普遍过量施用而导致成
本上升与环境污染的肥料。为了科学高效施肥，人 们迫切需要一种能准确、快速、方便、经济诊断作物 
氮素营养水平的方法。早在 1972 年 Thomas 等就发 现甜椒叶片含氮量与 $550 \sim 675 \mathrm{~nm}$ 波长间叶片的反 射系数高度相关, 实际含量与所预测的含氮量误差 小于 7\%(Thomas \& Oerther，1972），说明植物光谱分 析有可能快速、简便、较精确、非破坏性地监测植物 氮素营养。随后人们开始研究小麦氮素营养状况对 冠层光谱特性的影响 (Hinzman et al. , 1986) ,并探 讨了利用叶绿素计和冠层光谱来判断小麦氮素状况 的可行性(Reeves et al ., 1993 ; Filella et al .,1995)。 Fernandez 等 (1994) 发现用红光 (660 nm) 和绿光 (545 $\mathrm{nm})$ 两波段的线性组合可以预估小麦的氮含量。 Stone 等 (1996) 的研究表明植株氮光谱指数 PNSI (Plant nitrogen spectral index) 与小麦叶片氮吸收显著 相关, 不受生育时期的影响, 用 PNSI 来指导施肥能 显著改善氮素利用效率, 减少因过度施肥对环境造 成的污染。近年来, 随着高光谱遥感的兴起, 越来越 多的学者利用微分光谱和红边特性等来预测作物的 氮素状况 (Munden et al.，1994; 赵春江等，2001； Broge \& Mortensen , 2002)。但植物冠层反射光谱受 冠层几何结构、土壤覆盖度等因素的影响。由于不 同时空条件下这些影响因子不同,所建立的氮素光 谱诊断模型不能用于其建模以外的时空条件 (薛利) 红等 2003a)。迄今为止, 仍缺乏一个能准确定量评 价小麦氮素状况的非破坏性方法。为此, 本文选用 株型和叶色不同的两个代表性小麦品种, 系统分析 了不同时相下小麦叶片含氮量及叶片氮积累量与冠 层光谱反射特征的关系, 以期建立一个不受生育时 期和品种影响的通用小麦叶片氮素诊断模型, 为小 麦叶片氮素状况的精确诊断提供依据。

\section{1 材料和方法}

\section{1 试验设计}

试验在南京农业大学校内试验站的水泥池中进 行。土壤为黄棕壤土, 有机质 $1.21 \%$,全氮 $0.13 \%$, 速效氮 $29.2 \mathrm{mg} \mathrm{kg}^{-1}$, 速效磷 $29.5 \mathrm{mg} \mathrm{kg}{ }^{-1}$, 速效钾 $72.3 \mathrm{mg} \mathrm{kg}{ }^{-1}$ 。选用 2 个代表性小麦( Triticum aestivium)品种“: 徐州 26’ (叶色较浅, 叶片下披)和 淮 麦 18' (叶色较深, 叶片上冲) ;4 个氮肥水平 : NO , 不 施氮肥 , $\mathrm{N} 1,12 \mathrm{gN} \mathrm{m}^{-2}, \mathrm{~N} 2,21 \mathrm{gN} \mathrm{m}^{-2}, \mathrm{~N} 3,30 \mathrm{gN}$ $\mathrm{m}^{-2}$ 。小区面积 $4 \mathrm{~m}^{2}(2.4 \times 1.68 \mathrm{~m})$,两因素随机区 组排列,重复 3 次。氮肥的基追比为 $1:$, 追肥时间 为拔节期 (3 月 2 日)。磷、钾肥施用量分别为 $12 \mathrm{~g}$. $\mathrm{m}^{-2}\left(\mathrm{P}_{2} \mathrm{O}_{5}\right) 、 15 \mathrm{~g} \mathrm{~m}^{-2}\left(\mathrm{~K}_{2} \mathrm{O}\right)$,全部作基肥。基本苗 150 万. $\mathrm{hm}^{-2}$,行距 $24.5 \mathrm{~cm}$ 。其它栽培管理同大田
小麦高产栽培。

\section{2 光谱数据的测量及农学参数的测定}

采用美国 Cropscan 公司生产的 MSR-16 型便携 式多光谱辐射仪测量小麦群体光谱。分别在拔节 期、孕穗期、齐穗期、开花期、灌浆初期、灌浆中期、灌 浆后期进行测量。测量选择在晴朗无云的天气进 行测量时间为 $11: 00 \sim 14: 00$ 。测量时探头垂直向 下，距冠层顶部垂直高度约 $1 \sim 1.5 \mathrm{~m}$ 。每小区测量 3 点, 每点重复测量 5 次, 取平均值作为该小区光谱 测量值。

与光谱测量同步,每次每小区取代表性植株 5 $\sim 9$ 株, 测定和计算生物量 (鲜重、干重)、叶片全氮 含量、叶片氮积累量。氮的测定采用凯氏定氮法。 叶片氮积累量为单位土地面积上叶片所含的氮素总 量, 单位为 $\mathrm{g} \mathrm{m}^{-2}$ 等于叶片含氮量与单位土地面积 上叶片干重的乘积。

\section{3 数据分析}

光谱测量和农学参数的测量有时不能同步进 行, 甚至相隔 3 4 d 左右。为了修订这种不同步现 象, 可采用内插函数来计算光谱测量时的农学参数 (如叶面积指数等) (Broge \& Mortensen，2002)，或者 根据光谱反射率或植被指数随生育进程 (小麦用播 种后天数)的变化方程 (为二次曲线方程) 来推算农 学参数测量日期的光谱反射率或植被指数 (Gilabert et al. , 1996)。由于叶片含氮量等农学参数的变化 难以预测, 因此采用第二种方法来解决数据的不同 步问题。

传统的比值植被指数和归一化植被指数由于不 易受冠层结构等变化的影响而被经常用来预测叶绿 素含量，从而判断作物的氮素状况。为了确定能反 映小麦体内氮素状况的最佳宽波段光谱指数，本文 系统计算了从可见光波段到中红外波段共 16 个波 段的所有比值和归一化指数组合, 分析其与体内氮 素状况指标的相关关系。计算公式如下：

比值指数 : $R\left(\lambda_{1}, \lambda_{2}\right)=\rho_{\lambda_{1}} / \rho_{\lambda_{2}} ;$;归一化指数 : $N D\left(\lambda_{1}, \lambda_{2}\right)=\frac{\left|\rho_{\lambda_{1}}-\rho_{\lambda_{2}}\right|}{\rho_{\lambda_{1}}+\rho_{\lambda_{2}}}$

其中,$\rho$ 表示反射率, $\lambda$ 表示波段。

\section{2 结果与分析}

\section{1 不同小麦品种对氮肥的光谱响应}

从图 1 可以看出小麦冠层反射光谱的基本特 征 植物在可见光区的光谱特征受色素, 主要是叶绿 素 (还有类胡萝卜素等)的控制, 而叶绿素在可见光 


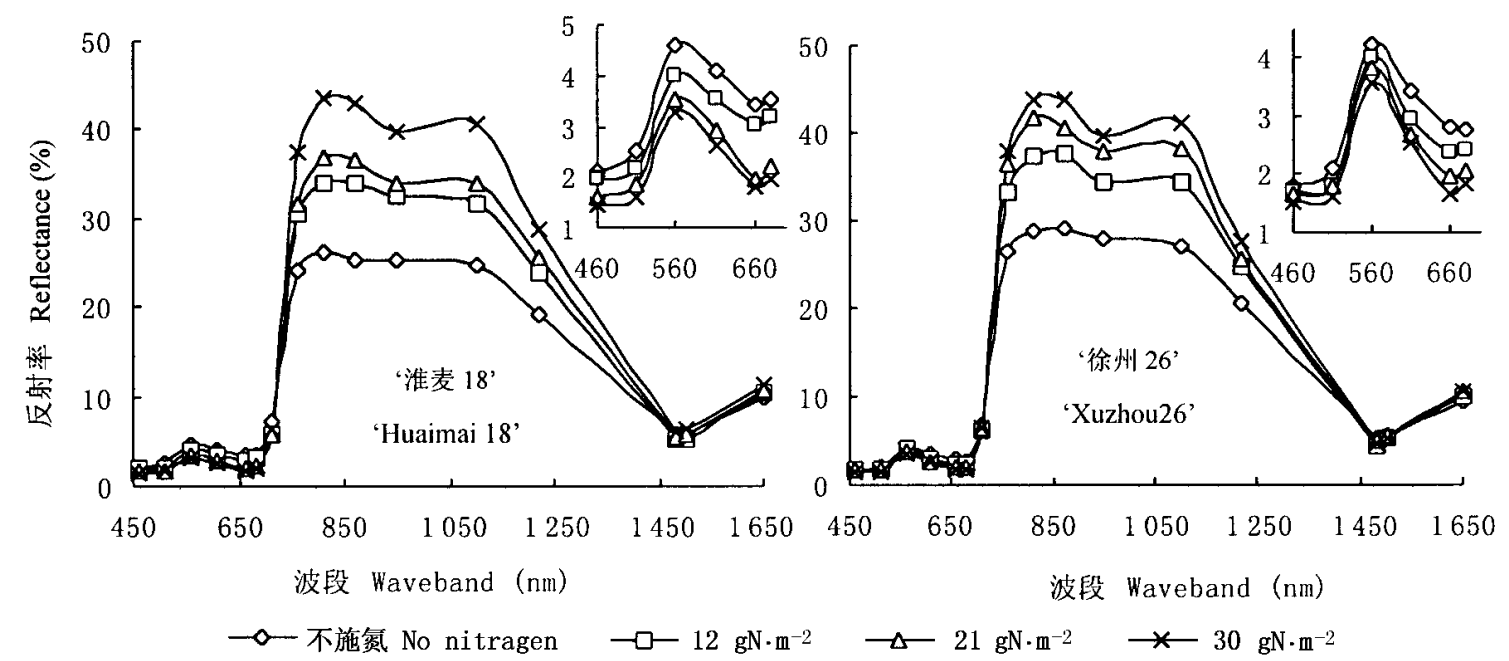

图 1 拔节期不同小麦品种冠层反射光谱对氮肥的响应模式

Fig. 1 The response pattern of canopy reflectance spectra of two wheat cultivars under different nitrogen levels at jointing stage

区有很强的吸光能力, 主要吸收蓝光和红光。因此 在 $680 \mathrm{~nm}$ 附近的红光区域和 $460 \mathrm{~nm}$ 的蓝光区有吸 收谷, 在位于蓝光和红光之间的 $550 \mathrm{~nm}$ 左右有反射 峰存在。在近红外区域由于受叶片结构的影响, 使 得 $810 \sim 1100 \mathrm{~nm}$ 波段出现一个较高的反射平台。 随施氮水平的增加，小麦冠层反射率在可见光区 $(460 \sim 710 \mathrm{~nm})$ 有所降低, 而在近红外波段明显升 高。两个小麦品种表现一致, 其它生育期测量的光 谱变化趋势也大体一致。这主要是因为增加施氮量 提高了叶片叶绿素含量、生物量和叶面积。叶绿素 含量的增加使对可见光波段大部分辐射的吸收增 强, 反射系数降低。在近红外波段则没有叶片色素 的吸收，所以，生物量和叶面积增加会增强散射返回 的概率，反射率从而增加。

\section{2 单波段反射率与小麦叶片氮素状况的关系}

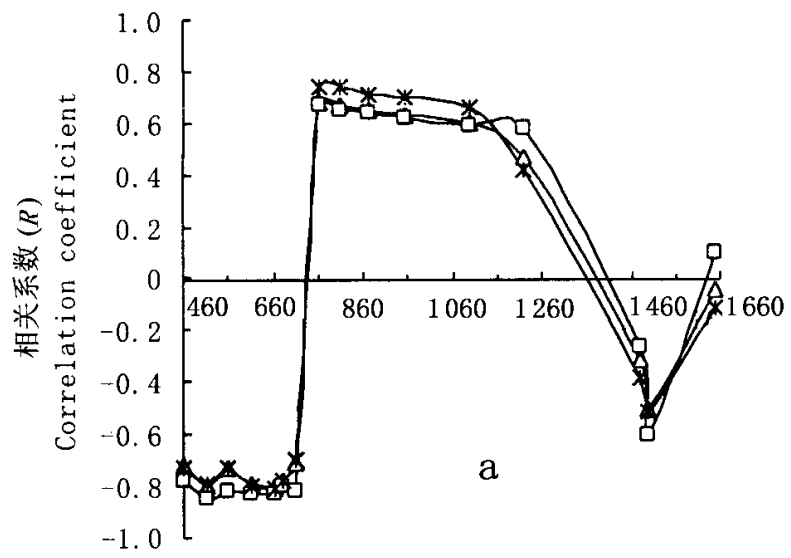

波段 Waveband (nm)

\section{2 .1 与叶片含氮量的关系}

对全生育期所有数据的相关分析表明，叶片含 氮量与可见光波段 ( $460 \sim 710 \mathrm{~nm}$ ) 和中红外波段 (1480 1 $650 \mathrm{~nm}$ ) 负相关, 与近红外波段 (710 $1220 \mathrm{~nm}$ )正相关，除了 $1650 \mathrm{~nm}$ 波段外，其余波段 都达到了显著水平，其中可见光波段的相关系数高 于近红外波段(图 2a)。两个品种之间差别不大, 在 可见光波段 淮麦 $18^{\prime}$ 的相关系数稍微高于 徐州 $26^{\prime}$,在近红外波段却略低于 徐州 $26^{\prime}$ 。当把两个 品种的数据放在一起分析时, 相关系数并没有明显 提高, 与单个品种的相关系数几乎持平。

对不同生育时期的数据单独进行分析时, 发现 无论是单个品种还是两个品种，近红外波段的相关 性在任一个生育阶段均高于可见光波段的，其中以 $610 \mathrm{~nm} 、 660 \mathrm{~nm} 、 680 \mathrm{~nm}$ 及近红外波段 (760 1 100

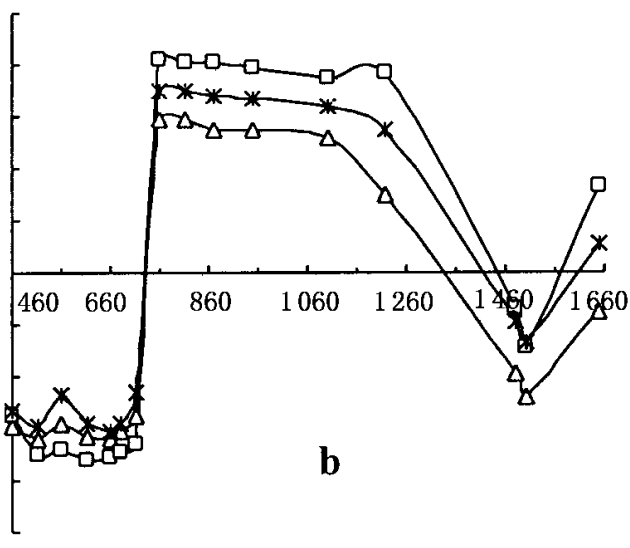

波段 Waveband (nm)

$\rightarrow$ 一两品种 Two cultivars 一- '淮麦18' 'Huaimai 18' —— '徐州26' 'Xuzhou 26' 
$\mathrm{nm}$ )的相关性最高。对 淮麦 18 ' 而言, 除了灌浆中 后期和孕穗期外，其余几个生育时期的相关性都较 高。而对 徐州 26'而言, 可见光波段的相关性较不 稳定, 近红外波段以抽穗期最好, 相关系数高达 0.80 。两个品种混在一起的相关性也以抽穗期最好， 其次为孕穗期 (数据未列出)。

\subsection{2 与叶片氮积累量的关系}

小麦叶片氮积累量与单波段反射率的关系同小 麦叶片含氮量。区别在于 徐州 26' 叶片氮积累量 的相关系数在所有波段都低于叶片含氮量;而 淮麦 $18^{\prime}$ 及两个品种组合叶片氮积累量的相关系数在可 见光波段低于叶片含氮量，在近红外波段刚好相反， 高于叶片含氮量(图 2b)。

单个生育时期的数据分析结果表明“, 淮麦 18 ’ 在大多数波段的相关系数在所有时期均达到了显著 水平;而 徐州 26 '在可见光波段的相关系数仅在蜡 熟期达到显著水平，在近红外波段除了灌浆中后期 外，其余都达到了显著水平。两个品种综合分析的 相关系数几乎都低于 淮麦 18 ', 但高于 徐州 $26^{\prime}$ 。 其中可见光波段表现不稳定 近红外波段表现较好， 在所有时期均达到显著水平, 以抽穗期为最佳(数据 末列出 )。

2.3 比值指数及归一化指数与小麦叶片氮素状况 的关系

\subsection{1 与叶片含氮量的关系}

在任一生育阶段，叶片含氮量与比值和归一化 指数均呈显著的正相关关系, 且两个品种表现极其 一致,可以用一个方程来拟合 (表 1)。其中开花期 表现最好, 决定系数在 0.80 以上, 其次为黄熟期和 抽穗期。此外, 与叶片含氮量相关性最好的比值指 数和归一化指数具有相同的波段组成。其中, 除了 灌浆中期 (4 月 29 日测) 为近红外波段 $(810 \mathrm{~nm}$ )与近 红边波段 $(710 \mathrm{~nm})$ 的组合外, 其余 6 个时期均为中
红外波段 (1 $500 \mathrm{~nm}$ 和 $1650 \mathrm{~nm}$ ) 与绿光波段 (560 $\mathrm{nm}$ )或近红外波段 (710 nm) 的组合。

对全生育期两个品种共 152 对数据综合分析， 发现叶片含氮量与比值指数呈显著的幂函数关系， 与归一化指数呈显著的指数函数关系 (图 3)。比值 指数中以 $R(660,460) 、 R(660,510)$ 及近红外波段 $(760 \sim 1220 \mathrm{~nm})$ 与 $660 \mathrm{~nm}$ 的比值为佳, $R^{2}$ 都在 0.80 以上, 其它近红外波段 (760 1220 nm) 与可见光波 段 $(460 \mathrm{~nm} 、 510 \mathrm{~nm} 、 560 \mathrm{~nm} 、 610 \mathrm{~nm}$ 和 $710 \mathrm{~nm}$ ) 的比 值及 $1650 \mathrm{~nm}$ 波段与 $510 \mathrm{~nm} 、 610 \mathrm{~nm} 、 660 \mathrm{~nm}$ 和 680 $\mathrm{nm}$ 波段的比值组合也都达到了极显著水平， $R^{2}$ 都 在 0.70 以上。归一化组合中以 $N D(660,460)$ 和 $N D$ (660 510) 最佳 $\left(R^{2}>0.80\right)$ ，其次为 $1650 \mathrm{~nm}$ 及近红 外波段 (760 1 $220 \mathrm{~nm}$ ) 与可见光波段 (510 nm、560 $\mathrm{nm} 、 610 \mathrm{~nm} 、 710 \mathrm{~nm})$ 的归一化组合、 $N D(660,560)$ 和 $N D(510460), R^{2}$ 也都在 0.70 以上, 达到了极显著 水平。

\subsection{2 与叶片氮积累量的关系}

不同生育时期叶片氮积累量与比值和归一化指 数也呈显著的正相关关系, 两个品种可以用一个统 一的方程来拟合。表 2 列出了最佳的比值和归一化 指数与叶片氮积累量的回归方程, 可以看出与叶片 氮积累量相关性最好的指数和与叶片含氮量相关性 好的指数极其类似, 也大多为中红外与可见光波段 的组合。不同之处在于与叶片氮积累量相关较特殊 的指数出现在拔节期, 为 $710 \mathrm{~nm}$ 与 $510 \mathrm{~nm}$ 波段的 组合。

叶片氮积累量与两波段的比值组合与归一化组 合显著正相关 (图 4)。对全生育期两个品种共 152 对数据一起分析，发现比值组合中，以 $R(1220,660)$ 最佳 $\left(R^{2}=0.72\right)$, 其次为近红外波段 (760 1220 $\mathrm{nm}$ )与可见光波段 $(460 \mathrm{~nm} 、 510 \mathrm{~nm} 、 610 \mathrm{~nm} 、 660 \mathrm{~nm}$ 、 $680 \mathrm{~nm})$ 的比值, $R^{2}$ 也都在 0.65 以上。在所有的归

表 1 不同生育时期两品种叶片含氮量与比值和归一化指数的回归方程

Table 1 The regression equations of leaf nitrogen concentration to ratio or normalized difference $(N D)$ index

\begin{tabular}{lcccccc}
\hline \multicolumn{1}{c}{$\begin{array}{c}\text { 生育时期 } \\
\text { Growth stage }\end{array}$} & $\begin{array}{c}\text { 比值指数 } \\
\text { Ratio index }\end{array}$ & $\begin{array}{c}\text { 回归方程 } \\
\text { Regression equation }\end{array}$ & $\begin{array}{c}\text { 决定系数 } \\
R^{2}\end{array}$ & $\begin{array}{c}\text { 归一化指数 } \\
N D \text { index }\end{array}$ & $\begin{array}{c}\text { 回归方程 } \\
\text { Regression equation }\end{array}$ & $\begin{array}{c}\text { 决定系数 } \\
R^{2}\end{array}$ \\
\hline 拔节期 Jointing & $R(1500,560)$ & $y=0.6645 x^{0.5302}$ & 0.5440 & $N D(1500,560)$ & $y=0.0149 x^{1.6962}$ & 0.6200 \\
孕穗期 Booting & $R(1500,560)$ & $y=0.8882 x^{0.5276}$ & 0.5979 & $N D(1500560)$ & $y=0.0836 x^{0.9081}$ & 0.6207 \\
抽穗期 Heading & $R(1500,710)$ & $y=3.2548 x^{1.1063}$ & 0.7190 & $N D(1500,710)$ & $y=7.8946 x+3.2677$ & 0.7082 \\
开花期 Anthesis & $R(1650,560)$ & $y=1.2622 x^{0.6465}$ & 0.8205 & $N D(1650560)$ & $y=0.2186 x^{0.6267}$ & 0.8227 \\
灌浆初期 Initial filling & $R(1100,710)$ & $y=1.6671 x+1.8819$ & 0.6829 & $N D(1100,710)$ & $y=0.0561 x+0.5754$ & 0.6799 \\
灌浆中期 Mid-filling & $R(810,710)$ & $y=2.2546 x-0.4994$ & 0.6394 & $N D(810,710)$ & $y=0.1028 x+0.4128$ & 0.6191 \\
灌浆末期 Late filling & $R(1500,510)$ & $y=0.8646 x+1.1617$ & 0.7461 & $N D(1500510)$ & $y=0.1332 x+0.2186$ & 0.7452 \\
\hline
\end{tabular}




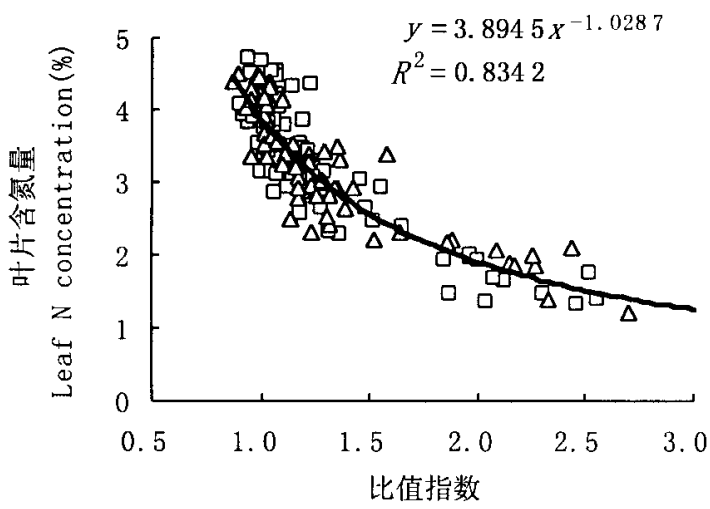

Ratio index of $660 \mathrm{~nm}$ and $460 \mathrm{~nm}$

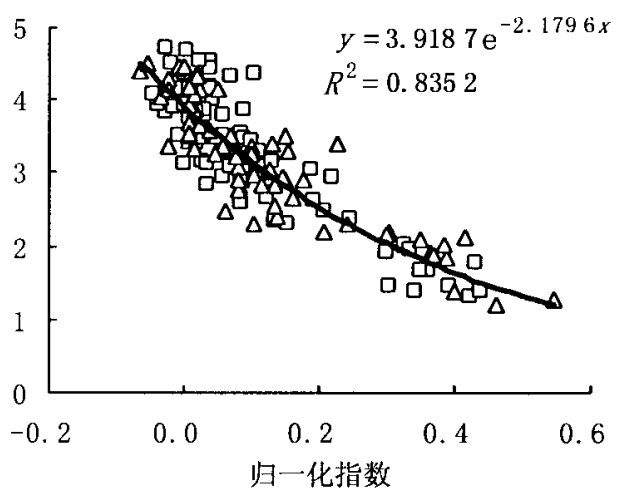

Normalized difference index of $660 \mathrm{~nm}$ and $460 \mathrm{~nm}$

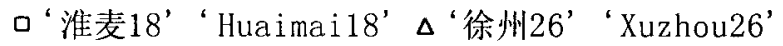

图 3 叶片含氮量与比值指数 $(R(660,460))$ 及归一化指数 $(N D(660,460))$ 的关系

Fig. 3 Relationship of leaf $\mathrm{N}$ concentration to ratio index and normalized difference index of $660 \mathrm{~nm}$ and $460 \mathrm{~nm}$

表 2 不同生育时期叶片氮积累量与比值和归一化指数的相关方程

Table 2 The regression equations of leaf nitrogen accumulation to ratio or normalized difference $(N D)$ index

\begin{tabular}{lcccccc}
\hline \multicolumn{1}{c}{$\begin{array}{c}\text { 生育时期 } \\
\text { Growth stage }\end{array}$} & $\begin{array}{c}\text { 比值指数 } \\
\text { Ratio index }\end{array}$ & $\begin{array}{c}\text { 回归方程 } \\
\text { Regression equation }\end{array}$ & $\begin{array}{c}\text { 决定系数 } \\
R^{2}\end{array}$ & $\begin{array}{c}\text { 归一化指数 } \\
N D \text { index }\end{array}$ & $\begin{array}{c}\text { 回归方程 } \\
\text { Regression equation }\end{array}$ & $\begin{array}{c}\text { 决定系数 } \\
R^{2}\end{array}$ \\
\hline 拔节期 Jointing & $R(710,510)$ & $y=0.054 x+2.7061$ & 0.7831 & $N D(710510)$ & $y=0.0061 x+0.4664$ & 0.7863 \\
孕穗期 Booting & $R(1480510)$ & $y=0.073 x+2.0382$ & 0.6849 & $N D(1480510)$ & $y=0.0099 x+0.3667$ & 0.6936 \\
抽穗期 Heading & $R(1500,610)$ & $y=1.0661 x^{2.0011}$ & 0.7356 & $N D(1500,610)$ & $y=37.581 x^{1.9824}$ & 0.7121 \\
开花期 Anthesis & $R(1650,610)$ & $y=0.4152 x^{2.1971}$ & 0.6816 & $N D(1650,610)$ & $y=43.121 x^{3.1277}$ & 0.6537 \\
灌浆初期 Initial-filling & $R(1100,680)$ & $y=0.8716 x+13.991$ & 0.7522 & $N D(1100,680)$ & $y=0.0045 x+0.8727$ & 0.7295 \\
灌浆中期 Mid-filling & $R(1220,560)$ & $y=0.3751 x+4.7132$ & 0.5747 & $N D(1220,560)$ & $y=0.014 x+0.6625$ & 0.5539 \\
灌浆末期 Late-filling & $R(1500460)$ & $y=3.1489 x^{0.195}$ & 0.7525 & $N D(1500 A 60)$ & $y=0.5215 x^{0.1117}$ & 0.7429 \\
\hline
\end{tabular}

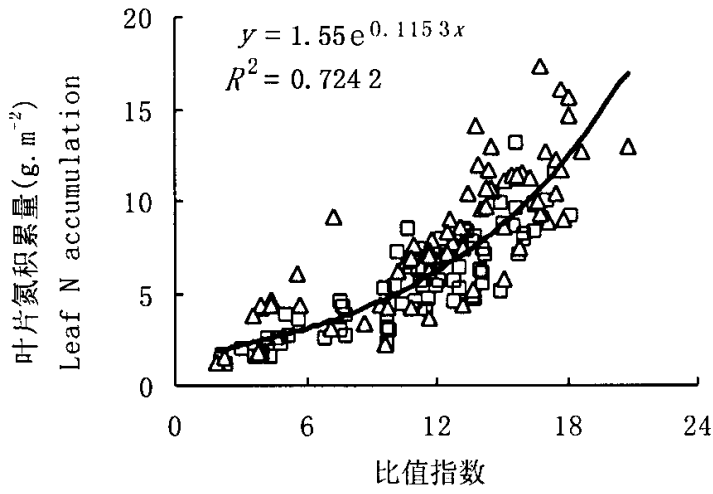

Ratio index of $1220 \mathrm{~nm}$ and $660 \mathrm{~nm}$

口“淮考18’ 'Huaimai 18'

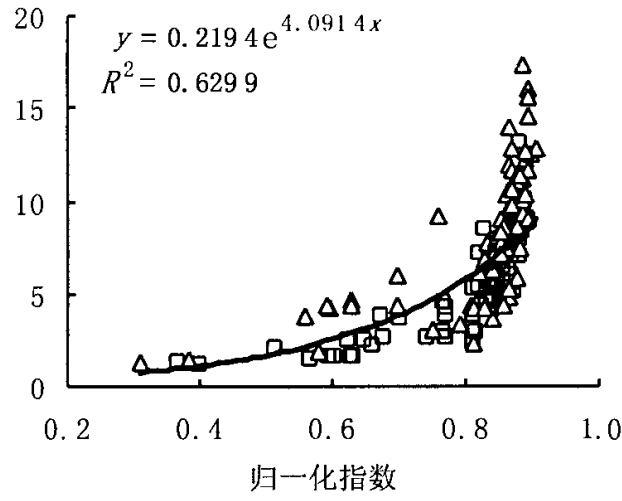

Normalized difference index of $1220 \mathrm{~nm}$ and $660 \mathrm{~nm}$

$\Delta$ “徐州26' 'Xuzhou26'

图 4 叶片氮积累量与比值指数 $(R(1220,660))$ 和归一化指数 $(N D(1220,660))$ 的关系

Fig.4 Relationship of leaf $\mathrm{N}$ accumulation to ratio index and normalized difference index of $1220 \mathrm{~nm}$ and $660 \mathrm{~nm}$

一化组合中, 以 $N D(1220,660)$ 与 $N D(1220,510)$ 最 佳 其次为近红外波段 $(760 \sim 1220 \mathrm{~nm}$ )及 $660 \mathrm{~nm}$ 波 段与 $460 \mathrm{~nm}$ 和 $510 \mathrm{~nm}$ 的组合, 1650 nm 波段与 510 $\mathrm{nm} 、 560 \mathrm{~nm} 、 610 \mathrm{~nm} 、 660 \mathrm{~nm} 、 680 \mathrm{~nm}$ 波段的组合及
$710 \mathrm{~nm}$ 波段与 $610 \mathrm{~nm}$ 和 $660 \mathrm{~nm}$ 波段的组合。

\section{3 小结与讨论}

本研究表明, 与叶片氮素状况相关性最佳的比 
值指数和归一化指数在小麦的不同生育时期具有相 同的波段组成,多为一中红外波段与一可见光波段 的组合, 但与叶片含氮量相关最好的指数不同于与 叶片氮积累量的。单个生育时期最佳回归方程的决 定系数为 0.82 ,并不高于全生育期的决定系数, 说 明分生育时期建立回归模型并没有提高模型的精 度。因此, 可以建立一个统一的适用于全生育期的 小麦氮素监测模型。

全生育期叶片含氮量与比值指数呈极显著的幂 函数关系,与归一化组合呈极显著的指数函数关系， 两个小麦品种的趋势基本一致,可以用一个方程来 拟合 (图 3)。且最佳的比值指数和归一化指数都由 $660 \mathrm{~nm}$ 和 $460 \mathrm{~nm}$ 两波段组合而成。这两个指数与 Penuelas 等定义的色素简单比值指数 PSR (Pigment simple ratio)和归一化色素指数 NPCI ( Normalized difference pigment index) 有点类似 (原指数中用了 680 $\mathrm{nm}$ 和 $430 \mathrm{~nm}$ 两个波段) (Penuelas et al., 1993 ; 1994)。当作物缺氮时, 体内的叶绿素 a 含量相对降 低, 而类胡夢卜素含量相对增加, 因此类胡夢卜素和 叶绿素 $\mathrm{a}$ 比值的动态变化能很好地指示作物氮素状 况 (Penuelas et al. , 1993 ; Young \& Britton , 1990)。 而 NPCI 和 PSR 与类胡夢卜素和叶绿素 a 比值密切 相关(Penuelas et al. , 1993 ; 1994), ,因此可以间接地 来判断叶片氮素状况。

全生育期叶片氮积累量与比值指数和归一化指 数显著正相关, 最好的比值和归一化指数分别为 $R$ (1 220,660$)$ 和 $N D(1220,460)$ 。Hinzman 等 (1986) 也发现小麦叶片氮积累量与比值植被指数 (近红外 与红波段的比值)线性正相关, 但本文的研究结果表 明用指数函数拟合的效果要优于线性函数, 决定系 数由 0.65 提高到了 0.72 。水稻上的研究表明, 用叶 片氮积累量可以提高冠层光谱指数监测氮素状况的 精度(薛利红等, 2003b)，但本研究结果却表明，小 麦叶片氮积累量与冠层光谱指数的决定系数要低于 叶片含氮量。这可能是因为试验中部分处理的小麦 在后期出现倒伏, 生物量降低, 对叶片氮积累量的准 确计算有一定的影响。

本研究建立的小麦叶片氮素诊断模型, 不仅适 用于全生育期, 且不受品种的影响。但由于参试品 种仅有两个, 因此还有待于进一步的验证。总体上 看 本研究运用冠层光谱特征特别是比值指数可以 较好地监测和诊断小麦叶片氮素状况。

\section{参 考 文 献}

Broge, N.H. \& J. V. Mortensen. 2002. Deriving green crop area index and canopy chlorophyll density of winter wheat from spectral reflectance data. Remote Sensing of Environment, 81: $45 \sim 57$.

Fernandez, S., D. Vidal, E. Simon \& L. Sole-Sugranes. 1994. Radiometric characteristics of Triticium cv. Astral under water and nitrogen stress. International Journal of Remote Sensing, 15: $1867 \sim 1884$.

Filella, I., L. Serrano, J. Serra \& J. Penuelas. 1995. Evaluating wheat nitrogen status with canopy reflectance indices and discriminant analysis. Crop Science, 35: $1400 \sim 1405$.

Gilabert, M. A., S. Gandia \& J. Melia. 1996. Analyses of spectral-biophysical relationships for a corn canopy. Remote Sensing of Environment, 55:11 20 .

Hinzman, L.D., M.E. Bauer \& C.S.T. Daughtry. 1986. Effects of nitrogen fertilization on growth and reflectance characteristics of winter wheat. Remote Sensing of Environment, 19: $47 \sim 61$.

Munden, R., P. J. Curran \& J. A. Catt. 1994. The relationship between red edge and chlorophyll concentration in the broadbalk winter wheat experiment at Rothamsted. International Journal of Remote Sensing, 15: $705 \sim 709$.

Penuelas, J., J. A. Gamon, A. L. Fredeen, J. Merino \& C. B. Field. 1994. Reflectance indices associated with physiological changes in nitrogen and water-limited sunflower leaves. Remote Sensing of Environment, 48:135 146 .

Penuelas, J., J.A. Gamon, K. L. Griffin \& C. B. Field. 1993. Assessing community type, plant biomass, pigment composition and photosynthetic efficiency of aquatic vegetation from spectral reflectance. Remote Sensing of Environment, 46: 110 118 .

Reeves, D. V., P. L. Mask, C. W. Wood \& D. P. Delaney. 1993. Determination of wheat nitrogen status with a hand held chlorophyll meter: influences of management practices. Journal of Plant Nutrition, 16: $769 \sim 781$.

Stone, M.L., J. B. Solie, W. R. Raun, R.W. Whitney, S. L. Taylor \& J. D. Ringer. 1996. Use of spectral radiance for correcting in-season fertilizer nitrogen deficiencies in winter wheat. Transactions of the ASAE (American Society of Agricultural Engineering), 39: $1623 \sim 1631$.

Thomas, J.R. \& G.F. Oerther. 1972. Estimating nitrogen content of sweet pepper leaves by reflectance measurements. Agronomy Journal, 64: $11 \sim 13$.

Xue, L.H. (薛利红), W. H. Luo (罗卫红), W.X. Cao (曹 卫星) \& Y.C. Tian (田永超). 2003a. Research progress on the water and nitrogen detection using spectral reflectance. Journal of Remote Sensing (遥感学报), 7: 73 80. (in Chinese with English abstract)

Xue, L.H. (薛利红), W.X. Cao (曹卫星), W. H. Luo (罗 卫红), D. Jiang (姜东), Y.L. Meng (孟亚利) \& Y. Zhu (朱艳). 2003b. Diagnosis of nitrogen status in rice leaves with the canopy spectral reflectance. Scientia Agricultura Sinica (中 国农业科学), 36: $807 \sim 812$. (in Chinese with English abstract)

Young, A. \& G. Britton. 1990. Carotenoids and stress. In: Alscher, R.G. \& J.R. Cumming eds. Stress response in plants: adaptation and acclimation mechanisms. New York: Wiley-Liss, Inc. $87 \sim 112$.

Zhao, C. J. (赵春江)，W.J. Huang (黄文江)，J. H. Wang (王纪华), M.H. Yang (杨敏华) \& X. Z. Xue (薛绪掌). 2001. The red edge parameters of different wheat varieties under different fertilization and irrigation treatments. Scientia Agricultura Sinica (中国农业科学), 35: $980 \sim 987$. (in Chinese with English abstract) 